\title{
A Method for Determining the Nose Radius of an Unequal Nose Radius Multi-Point Threading Tool*
}

\author{
Chin-Nan CHEN**, Chin-Hsi LIAO** and Yunn-Shiuan LIAO** \\ ** Department of Mechanical Engineering, National Taiwan University, No. 1, Sec. 4, Roosevelt Road, \\ Taipei, 10617 Taiwan, R.O.C \\ E-mail: d95522022@ntu.edu.tw
}

\begin{abstract}
Thread cutting is one of the crucial machining procedures of mechanical processing. However, breakage of the nose of a threading tool is relatively common in fine-thread machining and directly related to the nose radius. The design concept of the unequal nose radius $\left(\mathrm{UNR}^{\mathbb{1}}\right)$ tool involves increasing the radii of the front teeth of a multi-point threading tool and maintaining the radius of the last tooth as the nose radius of the standard thread. This design can enhance the machining efficiency and remove burr. However, no method exists for determining the nose radius. Ideally, each tooth of a UNR multi-point threading tool should have approximately the same life span. Because the life of the tool is inversely proportional to the strain value, we proposed comparing the maximum strain value of each tooth to determine the nose radii. A recursive binary search was adopted for comparison. The best values were then gradually converged into a single solution to obtain the optimal nose radii combination. Considering a 3-point threading tool for example, the recursion results provided the optimal radii combination. The experiments confirmed that the machining efficiency of the 3-point threading tool was 15 times of that of a single-point threading tool.
\end{abstract}

Key words: Unequal Nose Radius, Multi-Point Threading Tool, Thread Cutting, Recursion, Strain Value, FEM

\section{Introduction}

Two thread machining methods exist, that is, roll forming and turning. Roll forming is typically applied to materials with inferior mechanical properties. Although the machining accuracy is comparatively worse, the forming speed is faster; thus, this method is suitable for mass production. By contrast, turning is more suitable for workpiece materials with superior mechanical properties, producing a lower quantity with higher accuracy.

Both Kyocera $^{(1)}$ and Sandvik ${ }^{(2)}$, tool makers, provide two types of tools for thread cutting, single-point threading tools and multi-point threading tools. Single-point threading tools are used for thread machining differing pitches. Multi-point threading tools consist of two or more identical teeth arranged according to pitch, and can only be used to machine a single-pitch thread. The advantages of multi-point threading tools are better surface processing, less burr, and a longer tool life. Two types of multi-point threading tools, those with equal-height teeth and unequal-height teeth, are currently provided by makers. Only the first tooth of an equal-height multi-point threading tool has the function of removing materials; the remaining teeth only eliminate burr, whereas all teeth of an unequal-height

(1) UNR: unequal nose radius

${ }^{*}$ Received 26 Nov., 2012 (No. 12-0490) [DOI: 10.1299/jamdsm.7.205]

Copyright $\odot 2013$ by JSME 
multi-point threading tool have the function of removing materials. However, this type of threading tool is only produced with the same nose radii; thus, nose breakages remain a common problem when cutting difficult materials, such as titanium alloy.

As commonly known, the larger the nose radius, the greater the nose strength and less likely breakage will occur. The design concept of UNR multi-point threading tools involves increasing the nose radii, but maintaining the last nose radius as the standard thread requirement. The only difference between teeth is the nose radius. The bottom shapes of each tooth are identical and each tooth is responsible for removing materials from a different area. Although the identical bottoms of the teeth do not remove materials, they eliminate burr. The experiment results confirmed that the design could eliminate burr, increase efficiency, and prolong the tool life.

\section{Proposed approach}

Figure 1 depicts the undeformed chip shapes for different types of threading tools in thread cutting. Figure 1(a) shows the area of removed material after the first pass of a single-point threading tool. Figure 1(b) shows the area removed for each pass after the second pass. Figures 1(c) and 1(d) display the difference in thread cutting between equal-height and unequal-height multi-point threading tool designs. Figure 1(c) shows an equal-height two-point threading tool in which only the first tooth is responsible for removing material and the second tooth is responsible for cleaning out burrs. Figure 1(d) illustrates an unequal-height two-point threading tool in which the two teeth each remove a different part of the material, the results of which are similar to the second pass of a single-point threading tool.

(a)

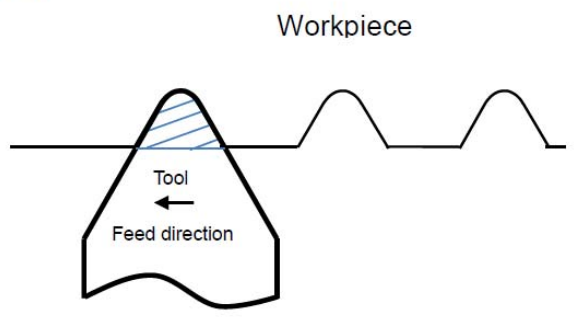

(c)

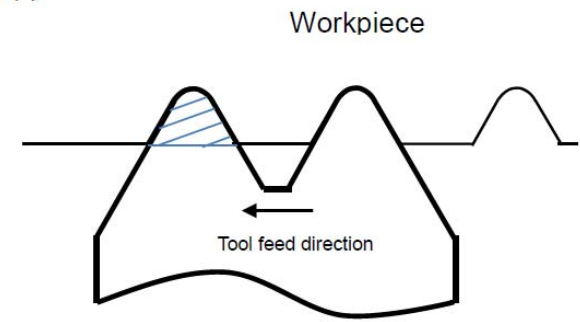

(b)

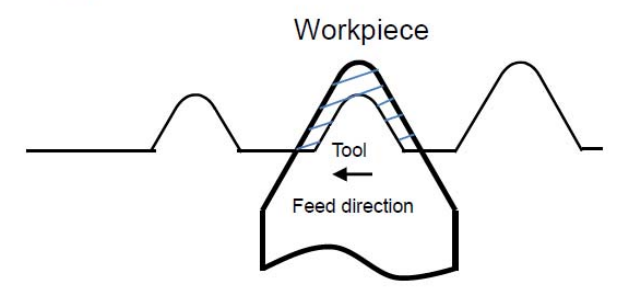

(d)

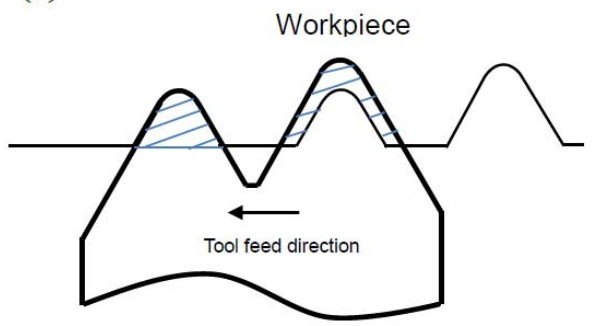

Fig. 1 The undeformed chip shapes: (a) first pass using single-point tool; (b) n-th pass using single-point tool, where $n>1$; (c) first pass using equal-height two-point tool; and (d) first pass using unequal-height two-point tool

The material removing condition of a UNR multi-point threading tool is shown in Fig. 2(a). The nose radii decreased gradually from the front to the rear. The shaded part denotes the area of each tooth that removes materials. By overlapping the shapes of three teeth, we found that the bottom shapes were identical, whereas the noses differed because of the varying radii. The material removing area of the teeth is shown in Fig. 2(b). 
(a)

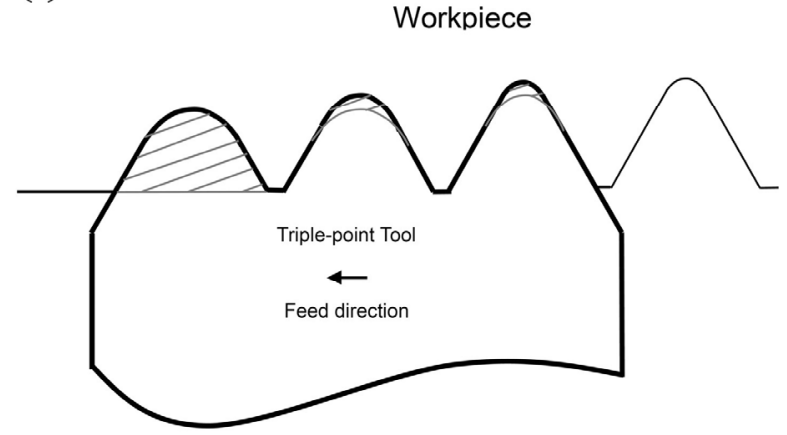

(b)

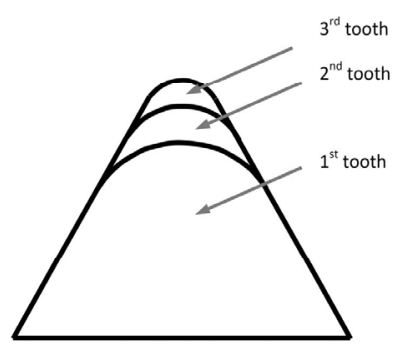

Fig.2 Thread cutting condition of a UNR 3-point threading tool: (a) the contact situation between cutters and materials; and (b) the shapes and thread-cutting areas of each tooth

Regarding determining the radius of each tooth, the main design concept was that the life of each tooth should be approximately the same, with the life of the front tooth slightly longer than that of the rear tooth. This design allows the thread to be completed regardless of whether the front teeth break during the thread-cutting machining process, providing the breakage is not serious. Zhou et al. ${ }^{(3)}$ highlighted that over $50 \%$ of tungsten carbide (WC) tool breakages are caused by fractures. He also used stress as an indicator to predict the life span of cutters. Therefore, in this study, tool life refers to unfixable breakages caused by fractures, not by wear.

Several studies have investigated tool fractures. Lee et al. ${ }^{(4)}$ predicted the mode and location of tool failures by conducting finite element method (FEM) analysis to examine the principal and von Mises stresses of the tool to determine the point of highest stress. Afaghani et al. ${ }^{(5)}$ showed that the relationship between the maximal stress and the number of impacts (stress cycles) required to rupture one block of tool grains have similar S-N fatigue curves. Antoniadis et al. ${ }^{(6)}$ developed an FEM simulation that can precisely calculate the stress strain field of a cutting tool. The model can be used to estimate the expected tool life and avoid early fractures of cutting tools. Thus, stress and strain are two of the most critical indicators for measuring tool failure. Furthermore, all studies have found that tool life is inversely proportional to stress and strain, and, as is well-documented, stress and strain are not only proportional to each other, but their ratio is also equal to the Young's modulus of the material. Because we recommended considering the material properties of the tool, in this study, strain value was used to calculate the tool life.

The studies mentioned previously indicate that tool life is inversely proportional to strain value. Thus, the strain value of each tooth can be the comparison basis for determining the nose radius of each tooth. To ensure that the life of the rear tooth is slightly longer than that of the front tooth, the strain value of the rear tooth must be less than that of the front tooth. To describe the relationship between the strain values of the rear and front teeth, we defined $d$ as the strain decreasing ratio of the rear tooth to the front tooth. Thus, to ensure that the life of the rear tooth is longer than the front tooth, $d$ must be smaller than 1 .

To determine the radius of each tooth of the multi-point threading tool, we adopted a recursive binary $\operatorname{search}^{(7)}$ to calculate all nose radii and strain values to satisfy the requirement of $d$ (strain decreasing ratio), and then gradually converged the best values into a single solution to obtain the optimum nose radii combination. According to the algorithm proposed previously, we designed a 3-point threading tool for turning an ISO $60^{\circ}$ titanium alloy thread with a $0.5-\mathrm{mm}$ pitch. The 3 -point threading tool was manufactured with three calculated radii and tested in actual cutting experiments. 


\section{Algorithm}

A temporary radius was determined for the first tooth as an initial value, and then the maximal strain value of the first tooth was calculated. Subsequently, the maximal strain value of the second tooth was calculated according to this maximal strain value and $d$ (strain decreasing ratio). Based on the strain value of the second tooth, we calculated the radius of the second tooth. The radii of the remaining teeth were determined using analogy. The radius of the last tooth was required to equal the thread standard, $1 / 10$ of the pitch overall. If the calculated radius was larger than the standard, the radius of the first tooth was reduced with a binary search, and the radii of the other teeth must be recalculated using the method explained previously. If the calculated radius was smaller than the standard, the radius of the first tooth was enlarged with a binary search, and the radii of the other teeth must be recalculated using the method explained previously. Recursive calculations were performed until the radius of the last tooth satisfied the required thread standard. The variables used during the calculation process, and their definitions, are listed below.

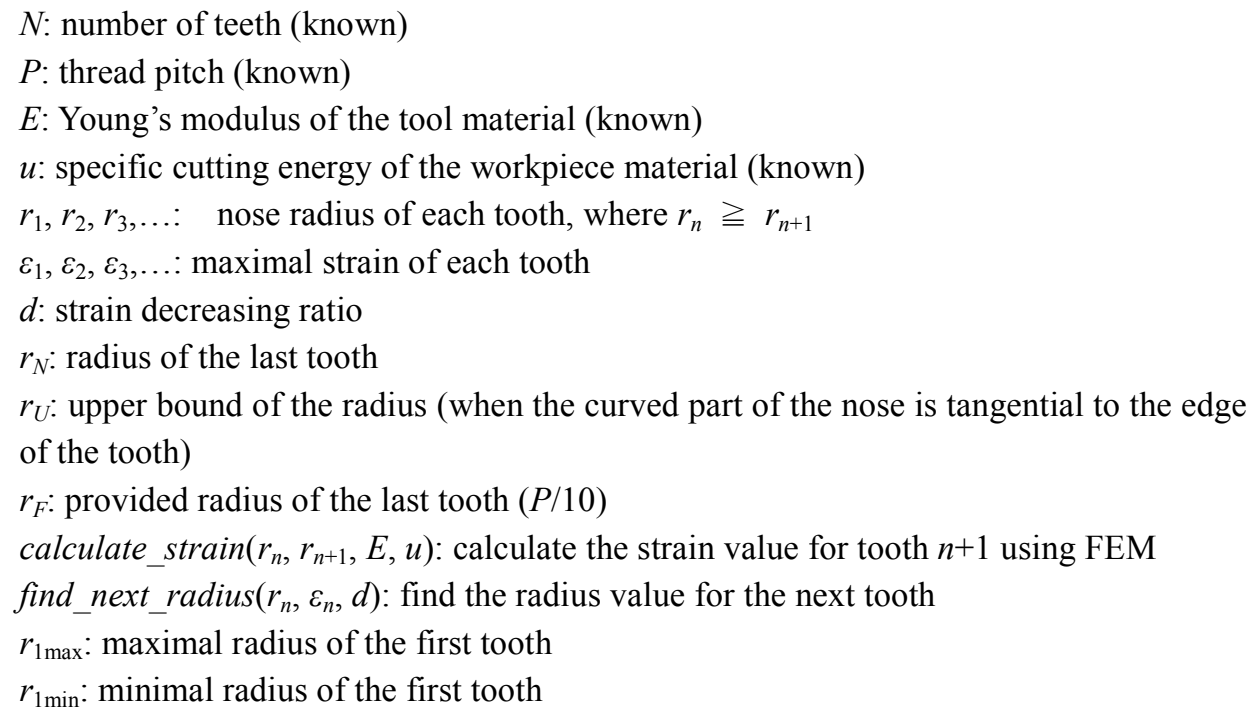

Regarding the function "calculate_strain $\left(r_{n}, r_{n+1}, E, u\right)$," FEM was used to calculate the state of strain when the $n+1$ tooth sustains cutting force. To calculate the strain of a tool under cutting force, the Young's modulus $E$ of the tool material, the tool shape, and the amount of force sustained by the tool are required. The force sustained by the tool equals the tool cutting force required to remove the materials and the specific cutting energy of the materials $u$, when represented by the unit area action. Therefore, the range of the material's undeformed chip area is the range of the force sustained by the tool when cutting. Additionally, the sustained force of the unit area equals the uniform strength of the workpiece material's specific cutting energy. Figure 3 shows the undeformed chip area of the $n+1$ tooth of the multi-point threading tool. 


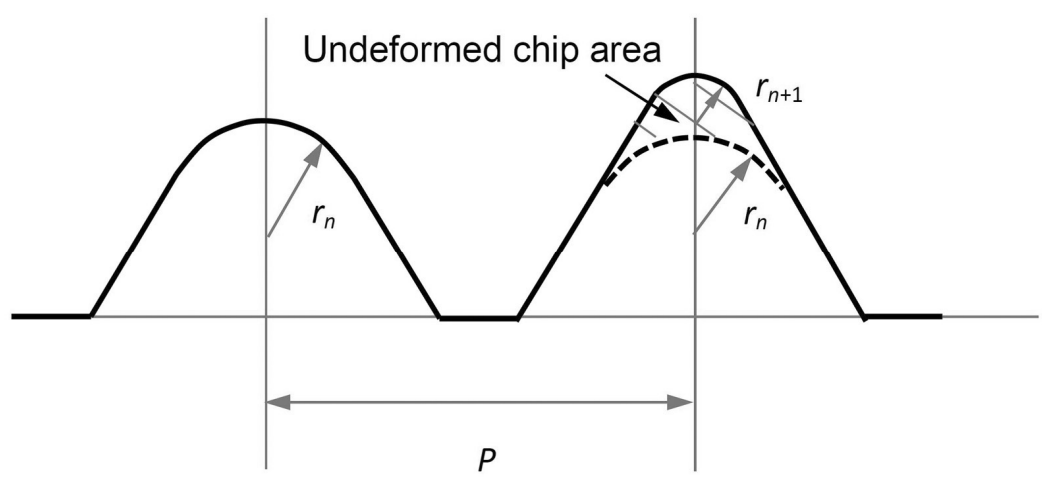

Fig. 3 The undeformed chip area of the $n+1$ tooth of the multi-point threading tool

As shown in Fig. 3, the undeformed chip area of the rear tooth is determined by the nose radius of the front and rear teeth. This means that if the radii of the front and rear teeth are determined, the strain value of the rear tooth can be calculated. Figure 4 shows the strain of the 3-point threading tool under force, as calculated by Solidworks with Cosmos FEM.

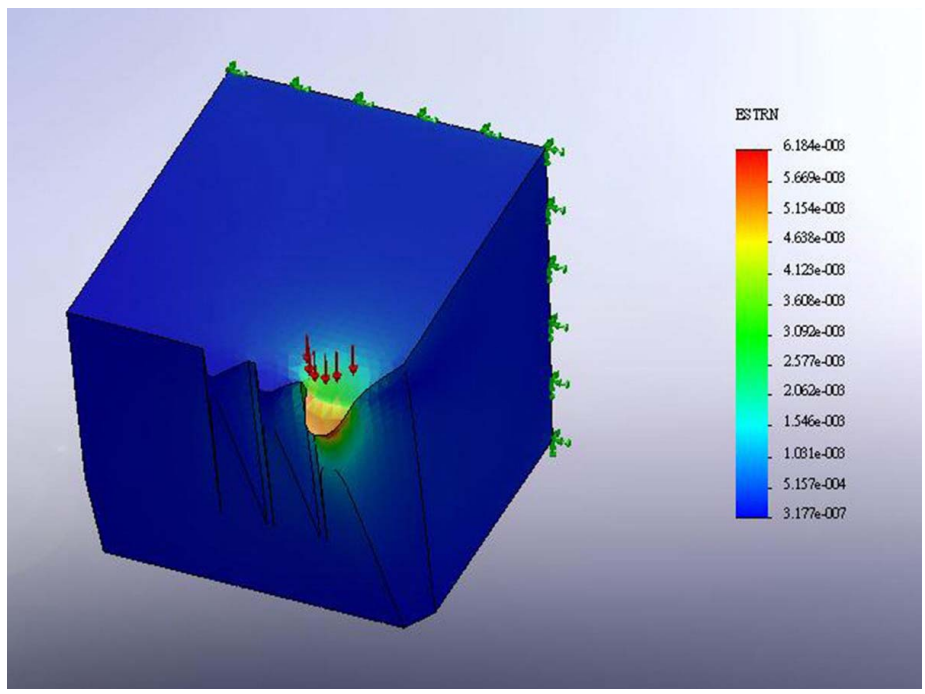

Fig.4 The strain of the 3-point threading tool under force, as calculated by Solidworks with Cosmos FEM

Table 1 shows the maximum stresses, maximum displacements and maximum strains of three-point threading tools, commercially available multi-point and single-point tool. The commercially available multi-point and single-point tools have the same result because both of them have the same nose radii and same cutting depth in the process of one-pass threading.

Table 1

FEM results of each tooth for three types of UNR three-point threading tool and commercially available multi-point and single-point threading tool 


\begin{tabular}{lcccccccccc}
\hline Max Stress $\left(10^{9} \mathrm{~N} / \mathrm{m}^{2}\right)$ & 5.14 & 4.97 & 5.22 & 5.02 & 5.14 & 5.01 & 5.13 & 4.93 & 4.66 & 5.36 \\
Max displacement $\left(10^{-6} \mathrm{~mm}\right)$ & 5.16 & 4.47 & 3.74 & 6.73 & 2.46 & 2.22 & 7.95 & 3.11 & 1.42 & 11.40 \\
Max strain $\left(10^{-3}\right)$ & 5.86 & 5.53 & 6.07 & 6.01 & 5.20 & 5.36 & 6.18 & 5.43 & 4.73 & 6.23 \\
\hline
\end{tabular}

However, the radius of the rear tooth can be calculated by establishing the strain value of the rear tooth. The function "find_next_radius $\left(r_{n}, \varepsilon_{n}, d\right)$ " uses this relationship to determine the maximal strain value $\varepsilon_{n+1}$ of the next tooth based on the maximal strain value of the previous tooth $\varepsilon_{n}$ and the established $d$ (strain decreasing ratio), and then calculates the radius of the next tooth $r_{n+1}$ using the known radius of the previous tooth $r_{n}$ and the expected maximal strain value $\varepsilon_{n+1}$. The calculation process of $r_{n+1}=$ find_next_radius $\left(r_{n}, \varepsilon_{n}\right.$, d) is explained below.

Step 1: Establish the initial maximal and minimal nose radii for the next tooth. The initial maximal value equals the radius of the previous tooth and the minimal value is the radius required by the final thread standard.

$$
r_{\max }=r_{n} ; r_{\min }=r_{F}
$$

Step 2: Use the mean value as the radius of the next tooth.

$$
r_{n+1}=\left(r_{\max }+r_{\min }\right) / 2
$$

Step 3: Use $\mathrm{r}_{\mathrm{n}} \mathrm{r}_{\mathrm{n}+1}$ to calculate the maximal strain value of the next tooth.

$$
\varepsilon_{n+1}=\text { calculate_strain }\left(r_{n}, r_{n+1}, E, u\right)
$$

Step 4: If the maximal strain value is smaller than that of the previous tooth, reduce the radius of the next tooth.

$$
\text { If } \varepsilon_{n+1}<d \varepsilon_{n} \text { then } r_{\max }=r_{n+1} \text {; goto Step } 2 \text {. }
$$

Step 5: If the maximal strain value is larger than that of the previous tooth, increase the radius of the next tooth.

$$
\text { If } \varepsilon_{\mathrm{n}+1}>d \varepsilon_{n} \text { then } \mathrm{r}_{\min }=r_{n+1} \text {; goto Step } 2 \text {. }
$$

Step 6: If $\varepsilon_{\mathrm{n}}=\mathrm{d} \varepsilon_{\mathrm{n}+1}$, then the radius of the next tooth is returned.

Return $r_{n+1}$

These calculations employ the concept of a recursive binary search. If the radius of the previous tooth $r_{n}$ is known, the radius of the rear tooth must be between the radius of the previous tooth $r_{n}$ and the radius of the last tooth $r_{F}$. Thus, use the mean value of the two radii as the initial radius of the next tooth $r_{\mathrm{n}+1}$ and conduct recursive calculations. Calculate the strain value of the next tooth $\varepsilon_{n+1}$ and examine the results against the expected $d \varepsilon_{n}$. If the two are equal, the calculation is complete. If $\varepsilon_{n+1}$ is smaller than $d \varepsilon_{n}$, let $r_{m a x}=r_{n+1}$. If $\varepsilon_{n+1}$ is larger than $d \varepsilon_{n}$, let $r_{\text {min }}=r_{n+1}$. Then recalculate the radius $r_{n+1}$. Figure 5 is a flowchart of $r_{n+1}=$ find_next_radius $\left(r_{n}, \varepsilon_{n}, d\right)$. 


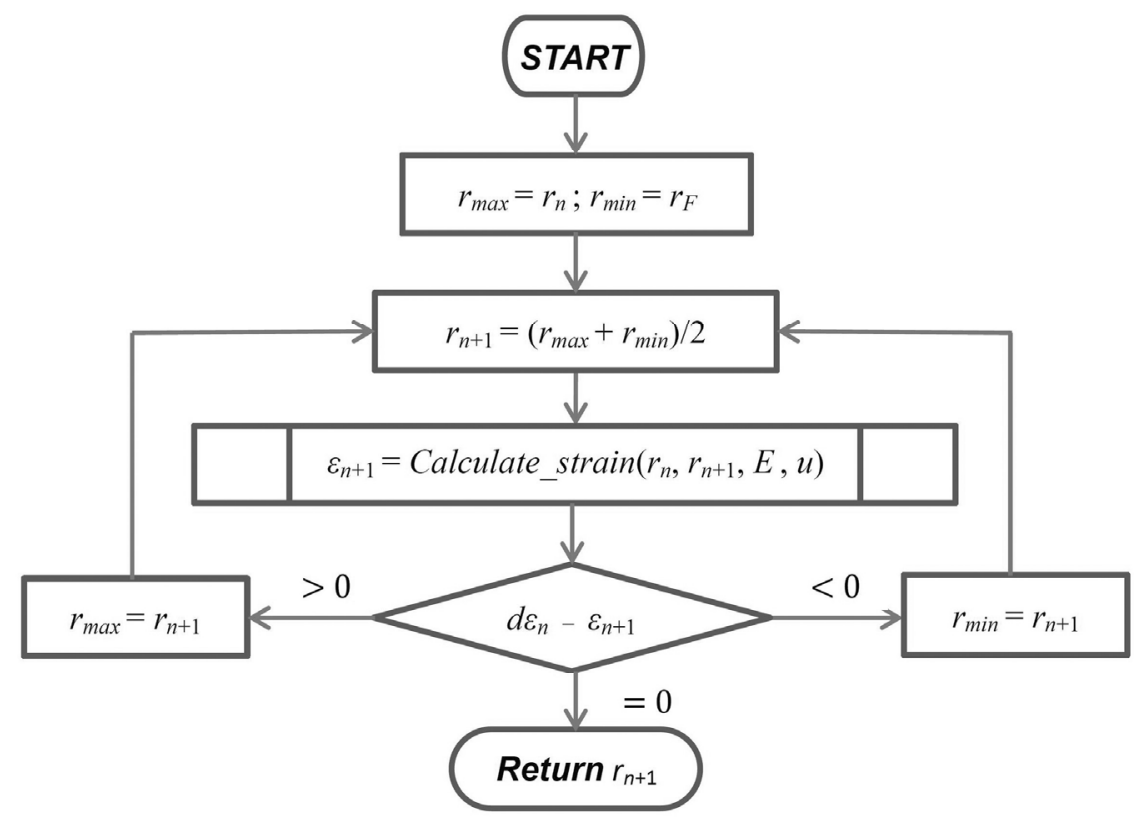

Fig.5 Flowchart of the function find_next_radius $\left(r_{n}, \varepsilon_{n}, d\right)$

If the radius of the first tooth $r_{1}$ is determined, using the function find_next_radius $\left(r_{n}, \varepsilon_{n}\right.$, $d$ ), the remaining radii can be calculated sequentially based on the first tooth. However, the radius of the first tooth is still unknown at the moment, here we apply recursive binary search calculations to determine $r_{1}$ as well. Let the maximum and minimum initial values of the first tooth nose radius be $r_{1 \max }$ and $r_{1 \min }$. Use the mean value of $r_{1 \max }$ and $r_{1 \min }$ as the initial value of the first tooth radius $r_{1}$ to calculate the maximal strain value of the first tooth by using function $\varepsilon_{1}=$ calculate_strain $\left(\infty, r_{1}, E, u\right)$, and then use find_next_radius $\left(r_{n}, \varepsilon_{n}, d\right)$ to calculate the nose radii of all other teeth. Finally, compare the calculated radius of the last tooth $r_{N}$ to the radius required by the standard thread $r_{F}$. If the two values are equal, the calculation is complete. If $r_{N}>r_{F}$, let $r_{1 \max }=r_{1}$. If $r_{N}<r_{F}$, let $r_{1 \min }=r_{1}$. Then recalculate $r_{1}$ and the nose radii of all other teeth. The flowchart of the calculations is shown in Fig. 6. 


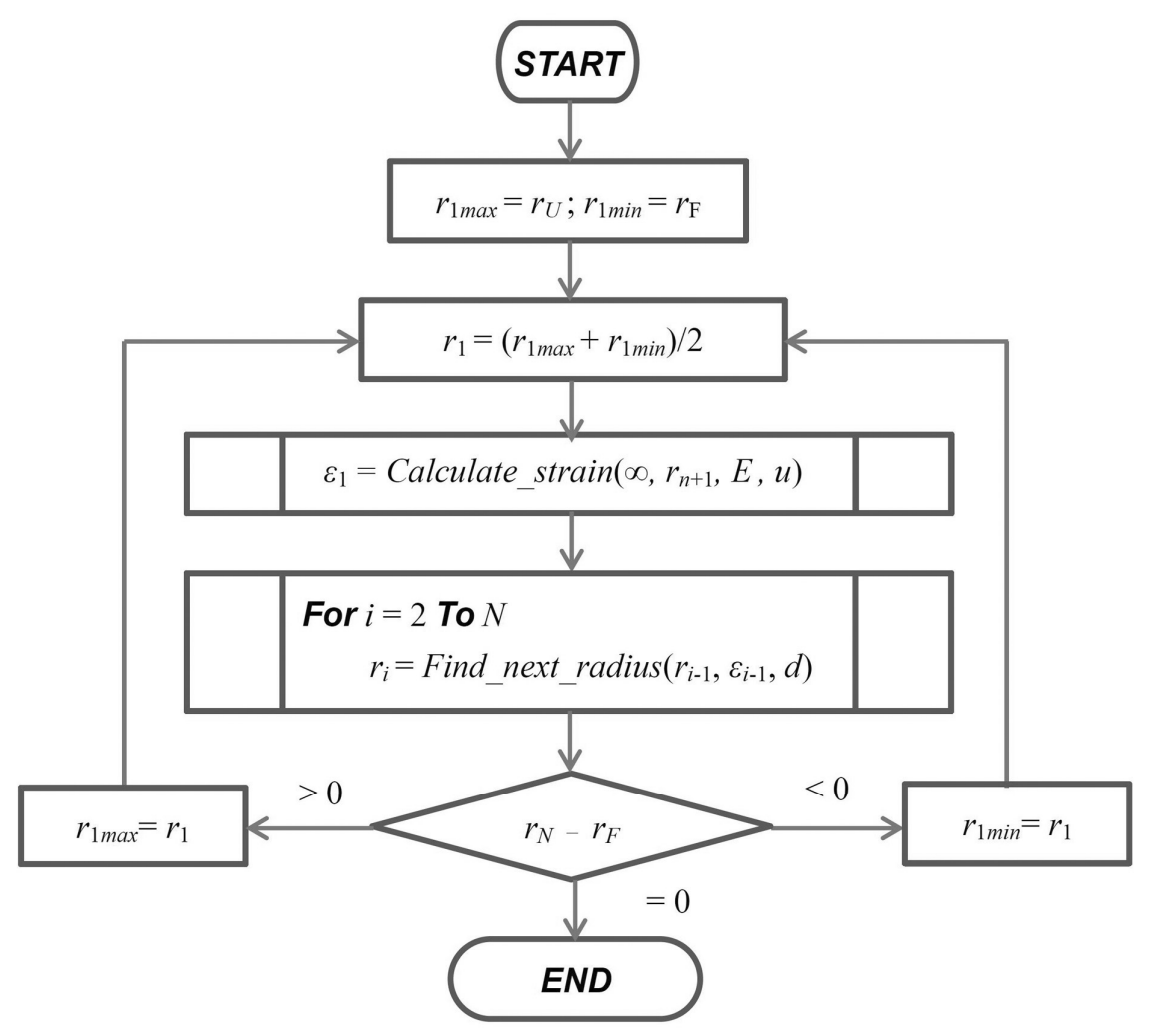

Fig.6 Flowchart of the process for determining the nose radius using binary search recursive calculations

\section{Experiments and Verification}

Many studies have pointed out that titanium alloy is difficult-to-cut material. Machado and Wallbank ${ }^{(8)}$ mentioned, when machining titanium and its alloys, the presence of high compressive stresses and also the development of high temperatures during catastrophic thermoplastic shear near the cutting edge cause plastic deformation at the cutting edge and/or at the tool nose, leading to tool breakage. Ezugwu and Wang ${ }^{(9)}$ demonstrated flank wear, crater wear, notch wear, chipping and catastrophic failure are the prominent failure modes when machining titanium alloys. Besides, according to Che-Haron ${ }^{(10)}$, straight tungsten carbide $(\mathrm{WC} / \mathrm{Co})$ cutting tools have proven their superiority in almost all machining processes. Venugopal et al. ${ }^{(11)}$ also mentioned that though ultra hard tool materials like cBN and PCD perform better than other tool materials, the high cost of such cutting tools compel the machining industries to look for other options. Straight tungsten carbide ( $\mathrm{WC} / \mathrm{Co}$ ) cutting tools perform best in machining of titanium alloys.

Therefore, for the experiments and verification, we used difficult-to-cut titanium alloy as the workpiece material and WC as the tool material to produce a 3-point threading tool for manufacturing ISO $60^{\circ}$ screws with a $0.5-\mathrm{mm}$ pitch to determine the nose radii of the three teeth. We adopted Solidworks and Cosmos FEM software for analysis. To simplify the analytical process, we conducted static analysis using the rear face of the tool as the fixed end and the uniform force of the front face. During the titanium alloy turning process, the cutting force acts on the front face. The cutting force was determined based on the well-known cutting force theory $F=u b t$, where $u$ represents the specific cutting energy of the titanium alloy, $b t$ is the undeformed chip area, and the cutting force per unit area equals the specific cutting energy $u$ of titanium alloy. Below we present the basic conditions of the analysis. 
Software : Solidworks 2008 and Cosmos 2008

Boundary conditions : rear face fixed

Loading : specific cutting energy of titanium alloy $\left(3.51 \times 10^{9} \mathrm{~N} / \mathrm{m}^{2}\right)$

Young's modulus of WC : $6.5 \times 10^{11} \mathrm{~Pa}$

Poisson ratio of $\mathrm{WC}: 0.28$

Analysis method : static analysis

The results indicated that the strain decreasing ratio $d=0.9$ because the cutting force was varied during the initial experiments using the single-point threading tool. The variation was maintained within $10 \%$ when cutting titanium alloy screws with a $0.5-\mathrm{mm}$ pitch. To ensure that variations of the strain value would not cause premature breakages of the rear tooth, the strain decreasing ratio of this experiment was determined to be $d=0.9$. The nose radius of the last tooth of an ISO $60^{\circ}$ screw with a $0.5-\mathrm{mm}$ pitch is typically $P / 10$. Therefore, the initial minimal value of the first tooth radius was $r_{1 \min }=0.05 \mathrm{~mm}$. The possible maximum radius of the first tooth $r_{U}$ was limited because the nose radius must be tangent to both sides of the tooth, as shown in Fig. 2(b). The maximal value of the first tooth for an ISO $60^{\circ}$ screw, which is the initial maximal value of the first tooth radius, was $r_{1 \max }=r_{U}=$ $P / 2=0.25 \mathrm{~mm}$. According to this calculation procedure, the known conditions were substituted to determine the radii of three teeth, that is, $0.15 \mathrm{~mm}, 0.08 \mathrm{~mm}$, and $0.05 \mathrm{~mm}$ for type I. To confirm the functionality of a triple-point UNR threading tool with this combination of radii, three additional tools with different nose radii were constructed for comparison. They are $0.15 \mathrm{~mm}, 0.1 \mathrm{~mm}$, and $0.05 \mathrm{~mm}$ for type II; $0.13 \mathrm{~mm}, 0.08 \mathrm{~mm}$, and $0.05 \mathrm{~mm}$ for type III; $0.1 \mathrm{~mm}, 0.1 \mathrm{~mm}$, and $0.05 \mathrm{~mm}$ for type IV which the first two teeth have different height. The actual shape of the front face and the tool is shown in Fig. 7. Both end relief angle and side relief angle are 15 degrees. 

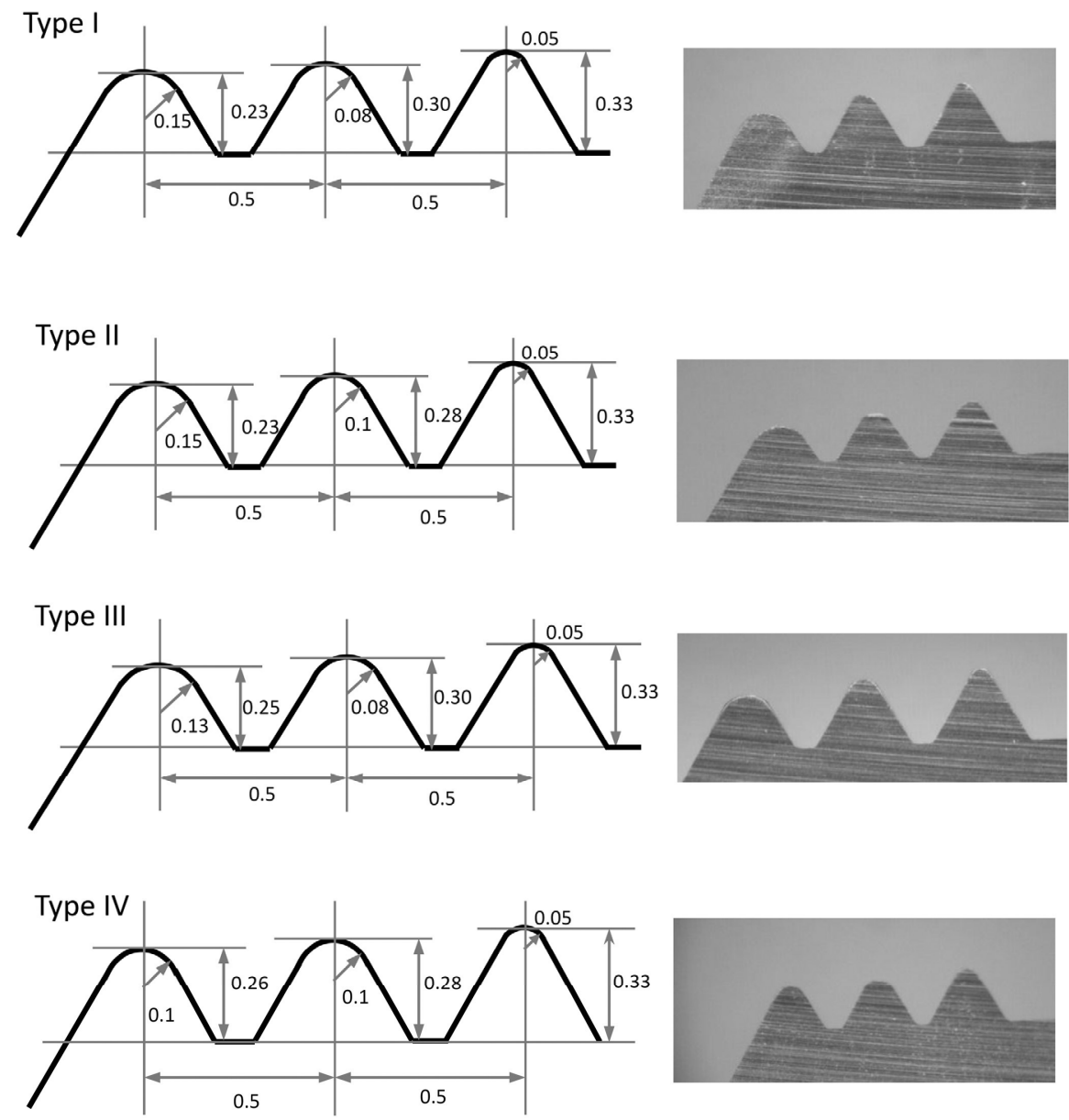

Fig.7 Design graph (unit: $\mathrm{mm}$ ) and actual picture of the four types of triple-point threading tools

Figure 8 shows the thread and tool state of the completed $50-\mathrm{mm}$-long $26.5 \mathrm{~mm}$ titanium alloy bars after thread machining on a $5 \mathrm{HP}$ lathe under $7 \mathrm{~kg} / \mathrm{cm}^{2}$ high-pressure air-cooling conditions. Figure $8(\mathrm{a})$ is a picture of Type I after it completed threading at a cutting speed of $58.2 \mathrm{~m} / \mathrm{min}$. This cutting speed is nearly three times faster than the 20 $\mathrm{m} / \mathrm{min}$ that a single-point threading tool with a $0.05-\mathrm{mm}$ nose radius can dry cut titanium alloy, and Type I did so without breaking. Thus, the efficiency of the UNR multi-point threading tool is 15 times higher. Figure 8(b) is a picture of Type II after its third tooth broke upon reaching $33.6 \mathrm{~m} / \mathrm{min}$. Figure $8(\mathrm{c})$ is a picture of Type III after its first tooth broke upon reaching $18.2 \mathrm{~m} / \mathrm{min}$. Figure $8(\mathrm{~d})$ is a picture of Type IV after its first tooth also broke upon reaching $20.1 \mathrm{~m} / \mathrm{min}$. 

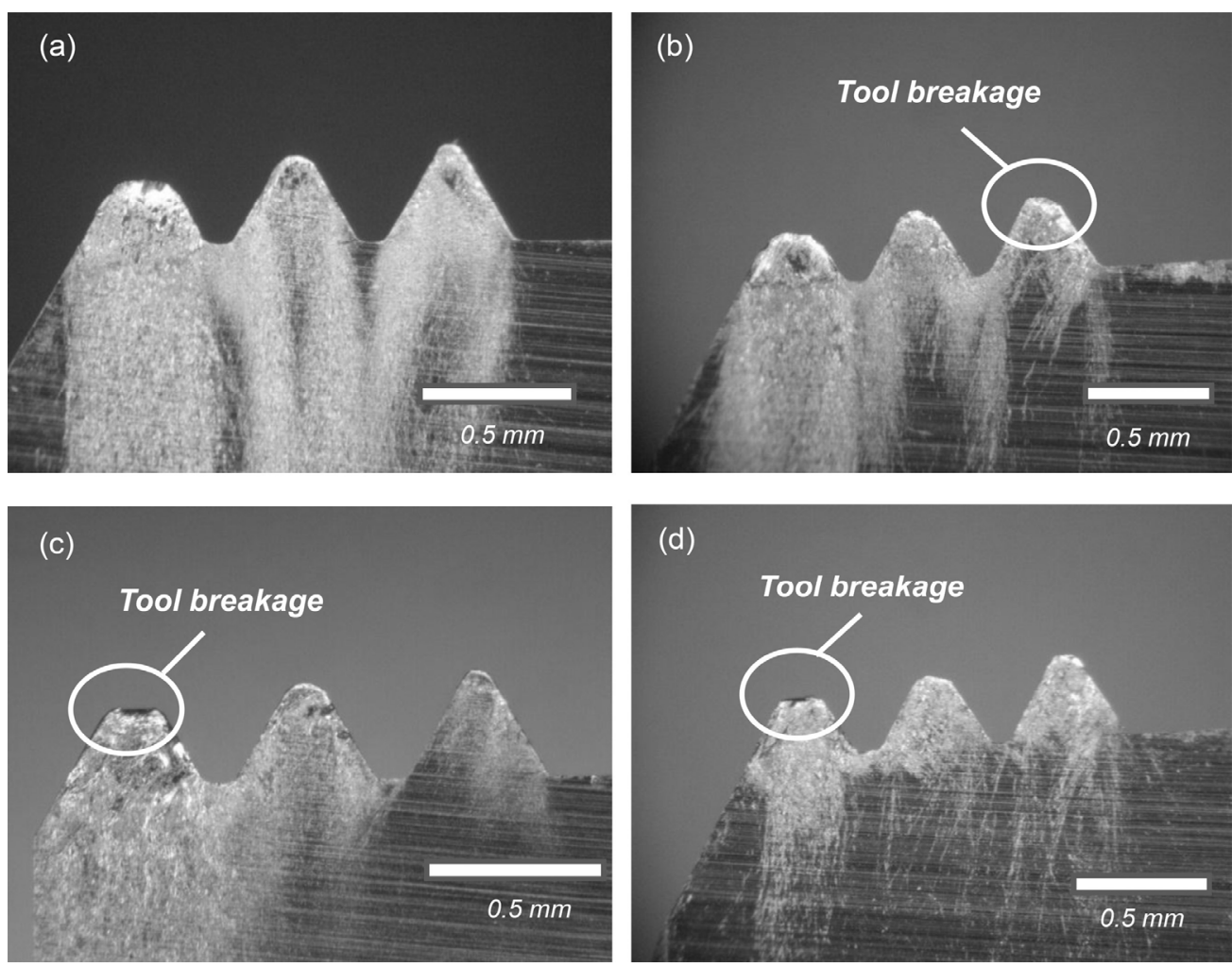

Fig.8 Experimental results of the four triple-point threading tool designs: (a) Type I at 58.2 $\mathrm{m} / \mathrm{min}$; (b) Type II at $33.6 \mathrm{~m} / \mathrm{min}$; (c) Type III at $18.2 \mathrm{~m} / \mathrm{min}$; and (d) Type IV at $20.1 \mathrm{~m} / \mathrm{min}$

Figure 9 shows a picture of the finished threading after one pass from the four different triple-point threading tools. As described before, the cutting speed gradually increased during the experiment. However, the tool was inspected after threading $50 \mathrm{~mm}$ of a titanium rod. The acceleration experiment halted once the tool was damaged. Therefore, excluding Type I, which could still thread at $58.2 \mathrm{~m} / \mathrm{min}$, the pictures of the other three tools were taken after tool damage was discovered following a completed segment of thread cutting. Figures 9(a), (b), (c), and (d) are the finished threading products at $58.2 \mathrm{~m} / \mathrm{min}$, $33.6 \mathrm{~m} / \mathrm{min}, 18.2 \mathrm{~m} / \mathrm{min}$, and $20.1 \mathrm{~m} / \mathrm{min}$, respectively. The damage to Type II was located in the third tooth. Figure 9(b) shows clear tooth markings at the base of the threading. Because the third tooth was not damaged in Type III and Type IV, the threading appears normal. However, when the cutting speed was increased to more than $30 \mathrm{~m} / \mathrm{min}$ to determine whether the third teeth in these two tools were able to remain in a usable state at higher speeds, the damage to the front teeth increased the cutting area covered by the back teeth, causing continuous damage to the subsequent teeth until it was no longer usable.

For comparing with the commercially available tools, the single-point tool experiments are conducted as well under the same cutting condition. Figure 10 shows the results of the tool face and thread surface after cutting. The breakages occurred on the single-point tool edge at cutting speed $18.0 \mathrm{~m} / \mathrm{min}$. The burr was observed on the thread surface. Evidently, the single-point tool was no longer usable. 

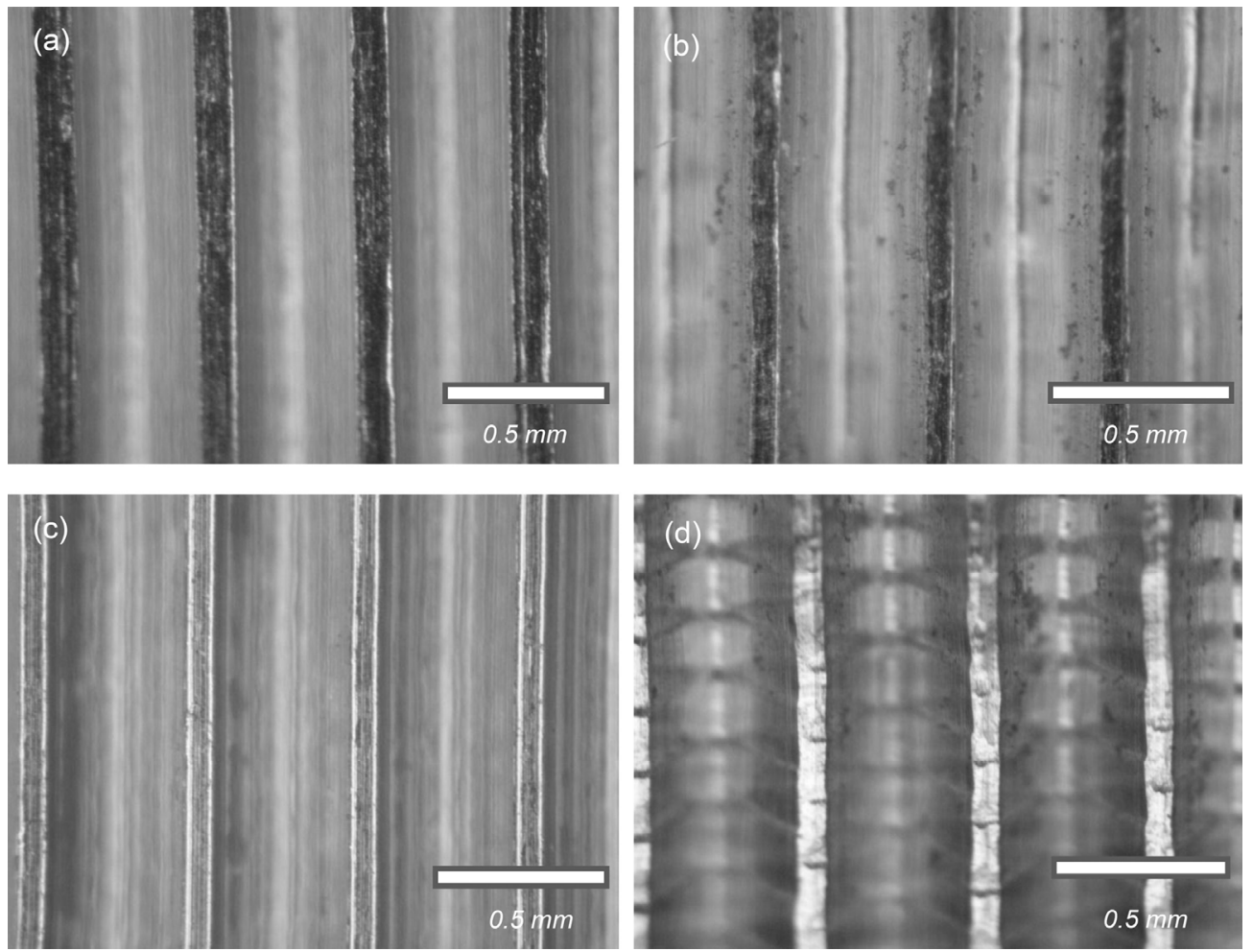

Fig.9 Pictures of thread cutting by the four different triple-point threading tool designs: (a) Type I at $58.2 \mathrm{~m} / \mathrm{min}$; (b) Type II at $33.6 \mathrm{~m} / \mathrm{min}$; (c) Type III at $18.2 \mathrm{~m} / \mathrm{min}$; and (d) Type IV at $20.1 \mathrm{~m} / \mathrm{min}$
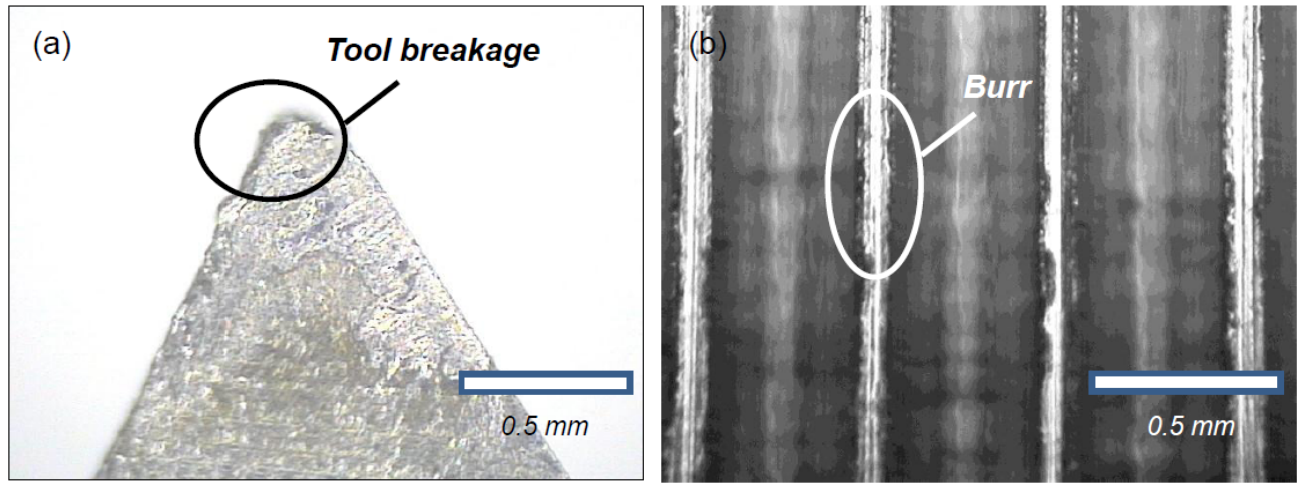

Fig.10 Experimental result of thread cutting by single-point threading tool at $18.0 \mathrm{~m} / \mathrm{min}$ (a) the photo of tool face; (b) the photo of thread surface

\section{Conclusion}

This study presents a method for a UNR multi-point threading tool, to determine the radius values for each tooth based on comparative strain values and using binary search recursive calculations. The following specific conclusions are drawn based on the results:

(1) As it is known, the larger unreformed chip area would cause the bigger cutting force on the tool, while the higher cutting speed generate more the heat. They are the two major factors causing the damage of the tools. However, when using the UNR tools, most of the material is removed by the first tooth, which has the biggest nose radius. On the other hand, the last tooth, which has the smallest nose radius and most vulnerable to damage, only remove small amount of the material. Hence even the 
cutting speed raises to three times higher than the normal speed, the process of threading can still be finished successfully.

(2) The multi-point threading tool current available commercially, either with equal depth of the teeth or not, comes with the same nose radius, which is, technically, with no difference to the single-point tool. Therefore the cutting speed could not be raised, and only the unequal height multi-point tool can finish the thread by less machining passes.

(3) Because FEM is used to calculate strain value, the strain value of cutters designed with any shape can be determined, and the optimal combination can then be identified through recursion. Therefore, this method can be applied to multi-point threading tools of various shapes; for example, cutters with differing nose angles or relief angles.

(4) If the workpiece material and the tool material are changed, the strain values can be recalculated by simply adjusting the Young's modulus and specific cutting energy values using FEM. The optimal combination can then be calculated through recursion.

(5) The strain decreasing ratio is determined according to the principle that the tool life of the rear tooth is slightly longer than that of the front tooth. An excessively small strain decreasing ratio indicates that the strain value of the front tooth is too high; this reduces the tool life of the front tooth and causes premature breakage. An excessively large strain decreasing ratio indicates that the strain value of the rear tooth is too high; this also reduces the tool life of the rear tooth and causes premature breakage. Thus, the optimum decreasing ratio can be determined according to the properties of the workpiece material and tool material or adjusted according to the breakage situation during actual machining.

(6) The algorithm proposed in this study can be used to determine the optimum combination of nose radii regardless of the tool shape, tool material, workpiece material, or differing teeth amount. Thus, the proposed algorithm can efficiently reduce the time required to design UNR multi-point threading tools.

\section{References}

(1) Kyocera Co., Cutting Tools catalogue, (2012), Kyocera Co.

(2) Sandvik Coromant, Main catalogue, (2012), Sandvik Coromant.

(3) Zhou, J.M., Andersson, M. and Sta ${ }^{\circ}$ hl, J.E., Cutting tool fracture prediction and strength evaluation by stress identification Part I: Stress, International Journal of Machine Tools and Manufacture, Vol. 37, No. 12(1997), pp.1691-1714.

(4) Lee, L.C., Lam, K.Y. and Liu, X.D., Characterisation of tool wear and failure, Journal of Materials Processing Technology, Vol. 40, No. 1-2(1994), pp.143-153.

(5) Afaghani, J.E.D., Yamaguchi, K., Nakamoto, T. and Yoshino, K., Tear-off wear mechanism of sintered diamond tool in cutting SiC-particle-strengthened epoxy composites, Wear, Vol. 206, No. 1-2(1997), pp.221-229.

(6) Antoniadis, A., Vidakis, N. and Bilalis, N., Fatigue fracture investigation of cemented carbide tools in gear hobbing, Part 1: FEM modeling of fly hobbing and computational interpretation of experimental results, Journal of Manufacturing Science and Engineering, Vol. 124, No. 4(2002), pp.784-791.

(7) H. Thomas, et al., Introduction to algorithms, (2009), MIT Press.

(8) Machado, A.R. and Wallbank, J., Machining of titanium and its alloys-a review, Proceedings of the Institution of Mechanical Engineers, Vol. 204, No. 1(1990), pp.53-60.

(9) Ezugwu, E.O. and Wang, Z.M., Titanium alloys and their machinability-a review, Journal of Materials Processing Technology, Vol. 68, No. 3(1997), pp.262-274. 
(10) Venugopal, K.A., Paul, S. and Chattopadhyay, A.B., Tool wear in cryogenic turning of Ti-6Al-4V alloy, Cryogenics, Vol. 47, No. 1 (2007), pp.12-18.

(11) Che-Haron, C.H., Tool life and surface integrity in turning titanium alloy, Journal of Materials Processing Technology, Vol. 118, No. 1-3(2001), pp.231-237. 